\title{
Evaluación de la gestión de cobro de la empresa ECONORTE, en el departamento de Estelí
}

María José Benavides Velásquez ${ }^{1}$

1 Correo Electrónico: cbmarynic@yahoo.es

\section{RESUMEN}

En esta investigación se determinan las malas prácticas utilizadas por los gestores de cobro de la empresa de cobros ECONORTE (Empresa de Cobros del Norte) que realizan su gestión de recuperación de cobreo en Estelí y sus municipios. El tipo de investigación que se realizó es descriptiva y analítica, con propuesta, se sustentó teóricamente en Beaugond, R. (1997), Carh, Pos. (2007), ttinger, R, P. (2000), Figueros, S (21 de Marzo- 2003), entre otros. Se aplicó un cuestionario de preguntas, dirigida tanto para el personal de la empresa como para los clientes que requieren del servicio que brinda la empresa. Los resultados evidencian que, según los gestores de cobro de Econorte las acciones y estrategias que siguen para la recuperación de la cuentas por cobrar de la empresa Disnorte son las correctas y se le esta dando el debido seguimiento a los clientes, además de estar en comunicación con los Distribuidores de Facturas para coordinar el cobro de las mismas.

Según la encuesta realizada a los clientes lo anterior no es del todo efectivo ya que no se le está realizando la visita mensualmente por lo que al parecer la gestión no es la correcta.

Palabras Claves: ECONORTE, Contrata, Gestor de cobro, Negociar, Gestor. 


\title{
Evaluation of collection management company ECONORTE, in the department of Estelí
}

María José Benavides Velásquez ${ }^{1}$

1 E-mail: cbmarynic@yahoo.es

\begin{abstract}
This research identifies the bad practices used by managers to collect the billing company Econorte (North Collection Company) performing recovery management cobreo in Estelí and its municipalities. The research that was done is descriptive and analytical, with proposal was based theoretically on Beaugond, R. (1997), CARH, Pos (2007), Ettinger, R, P. (2000), Figueros, S (March 21, 2003), among others. A questionnaire of questions addressed to the staff of both the company and for customers requiring the service provided by the company. The results show that, as managers Econorte collection actions and strategies followed for recovery of accounts receivable of the company Disnorte are right and he is giving up due to customers, as well as being in communication Bill with Distributors to coordinate the collection of the same.
\end{abstract}

According to the survey of customers above is not entirely effective because this is not conducting monthly visits so that management apparently is not correct.

Keywords: Econorte, hire,Collection Manager,negotiate, Manager. 


\section{INTRODUCCIÓN}

La gestión de cobro es un factor importante en una empresa de servicios, como es la empresa de cobro del norte S.A (ECONORTE), es una sociedad en la cual se forma con el objetivo de ofrecer servicios de cobro de facturación de energía, recuperación de mora y cualquier servicio semejante. Los buenos resultados que aporta un especialista en la recuperación de deudas muchas veces dependen de sus propias aptitudes y cualidades personales, es decir de la manera como hace las cosas y del modo de ser de la persona, y no de los medios tecnológicos de los que dispone el profesional para desarrollar sus tareas. Consecuentemente las cualidades y conocimientos del gestor de cobros son fundamentales para obtener buenos frutos en la gestión de cobranzas

\section{MATERIALES Y MÉTODOS}

El tipo de investigación que se realizó es descriptiva y analítica, con propuesta y descriptiva porque se describió la situación actual de la empresa y se analizan cuales son la debilidades que les afectan. Analítica porque abstrae el problema empresarial y lo explica en sus causas y consecuencias de la problemática actual Finalmente será con propuesta, porque se propondrán estrategias de mejora a utilizar para atender el problema planteado.

El diseño de esta investigación fue de tipo No experimental, se basó en información obtenida y generada, que constituyó en si misma las respuestas a las preguntas planteadas en el problema de investigación

La población en estudio se constituyó de la siguiente manera: Socios, gestores de cobro y 39,166 clientes de la cartera de Estelí en 2011 y la muestra está conformada por: 8 socios ya que hay un socio inactivo trabajadores de la empresa ECONORTE y 100 clientes de Estelí.
Las técnicas de procesamiento y análisis fueron: Técnica de la Entrevista. Se utilizara un cuestionario de preguntas, dirigida al presidente de la sociedad de la empresa de Cobro del Norte ECONORTE y la Técnica de la encuesta, consiste en un cuestionario de preguntas, dirigida tanto para el personal de la empresa como para los clientes que requieren del servicio que brinda la empresa y la Observación para corroborar como los trabajadores de la empresa realizan su trabajo.

\section{Análisis de Resultados}

Una vez procesado y analizada toda la información se llegó a los siguientes resultados. Para analizar la gestión de cobro se aplicaron una encuesta los clientes obteniendo los siguientes resultados:

Pregunta.1 ¿Desde cuándo es usted cliente de la empresa ECONORTE?

\section{Desde cuándo es usted cliente de la empresa ECONORTE}

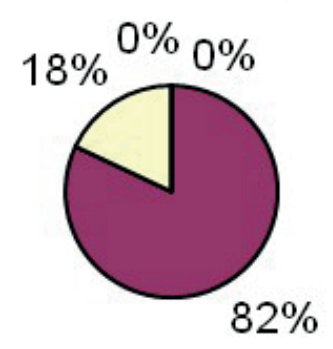

\begin{tabular}{|l|}
\hline Menos de un \\
año \\
घntre 1 - 3 \\
años \\
Entre 4 - 8 \\
años \\
Más de 9 \\
años \\
\hline
\end{tabular}

Como se puede observar en la tabla, se detectó que dentro de los 10 años que tiene la empresa ECONORTE (Empresa de cobros del norte) en funcionamiento los clientes tienen conocimiento de su existencia con $82 \%$ dentro de los 4 y 8 años y con un $18 \%$ entre 1 y 3 años.

Según Sibila Customer Intelligence ${ }^{\mathrm{TM}}$, solución avanzada para marketing y ventas, la captación de clientes a través de medios tradicionales y nuevos 
medios continuará siendo prioridad número uno para las áreas y responsables de marketing y ventas alrededor de todo el mundo en 2011.

Sibila Intelligence realizó recientemente un estudio, del que destacamos a continuación cinco consejos que podrían ser muy útiles: Incorporar, de una manera efectiva, las redes sociales como un canal más de captación, Segmentar los clientes potenciales para racionalizar inversiones, conocer y explotar las redes de influencia y relaciones entre los clientes, convertir las oportunidades de venta en experiencias de compra para el cliente, y en cualquier caso, escuchar a cliente. El resultado de la encuesta realizada a una muestra de 100 clientes demuestra que dentro de los 10 años que tiene la empresa ECONORTE (Empresa de cobros del norte) de ser contrata de Disnorte (Distribuidora de Energía del norte) la mayor captación de clientes la tuvo dentro de los primeros 8 años. Es claro y evidente que la empresa de Cobros del Norte (ECONORTE) no tiene ninguna estrategia para dar a conocer sus servicios, por lo que no gestionan deuda de nuevos a clientes.

Pregunta 2: ¿Cómo conoció el servicio de Econorte (Empresa de obro del Norte)?

\section{Como conocio el servicio}

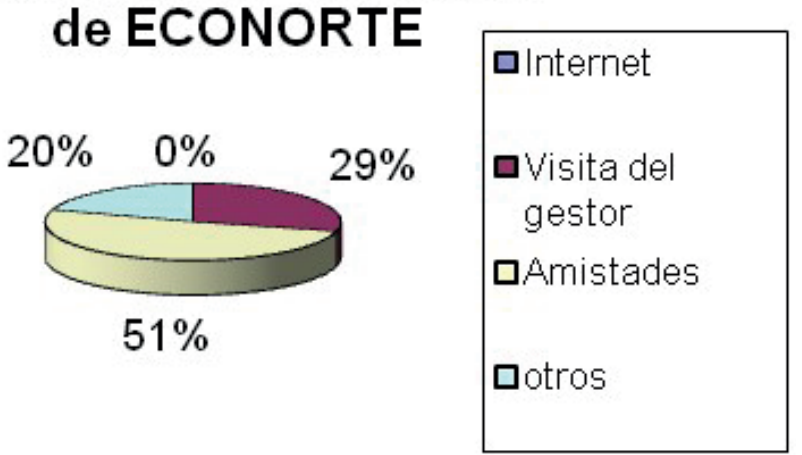

Al consultar con los clientes como conoció el servicio que brinda la empresa con un 29 \% Visitados por el gestor, un $51 \%$ información de amistades y con un $20 \%$ otros (Radio, Televisión, Volantes, Perifoneo, otros)
Según SALVADOR FIGUEROS el 30 JULIO, 2011 En uno de los elementos más importantes en cualquier negocio es contarle al mundo quién eres. Darte a conocer.

Si tu mercado no sabe que existes, no existes. A partir de ahí, no puedes hacer nada. Según Philip Kotler "La mejor publicidad es la que hacen los clientes satisfechos. "Con la encuesta realizada a los 100 clientes de ECONORTE, da como resultado que no tiene ninguna estrategia para dar a conocer su servicio, no cuentan con afiches, anuncios (tv, radio, internet), volantes entre otros, por lo que únicamente los clientes se dan cuenta de su existencia mediante comentarios de amistades, vecinos etc.

Pregunta 3: ¿Utiliza todos los meses el servicio del gestor?

\section{Utiliza todos los meses el servicio del gestor?}

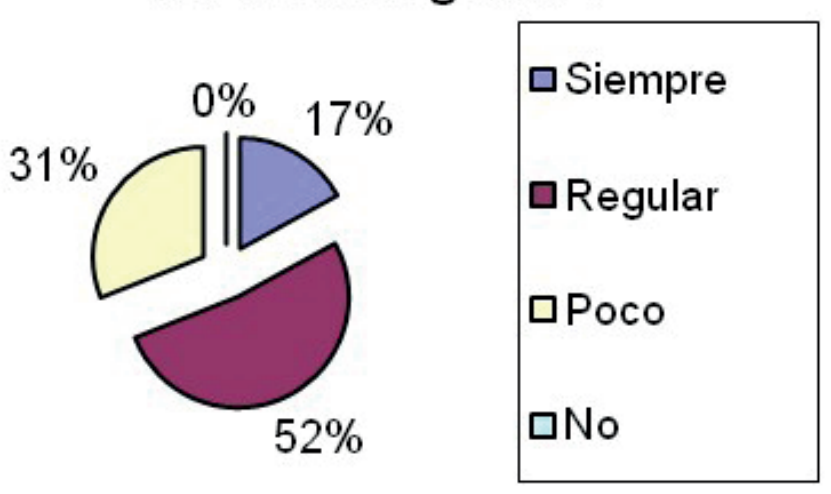

También se consultó a los clientes si utilizan todos los meses el servicio y el $17 \%$ dijo que siempre lo utiliza, el $52 \%$ dijo que no siempre y el $31 \%$ dijo que poco. Los resultados que arroja esta pregunta realizada a los clientes que utilizan este servicio nos demuestra que los gestores no le dan seguimiento al cliente mensualmente y analizando el cobro por localidad me doy cuenta que algunas localidades de la zona rural no son visitadas mensualmente si no mes de por medio. 
Pregunta 4: ¿Cuál es el grado de satisfacción del servicio que le brinda el gestor?

\section{Cuál es el grado de satisfacción del servicio que le brinda el gestor}

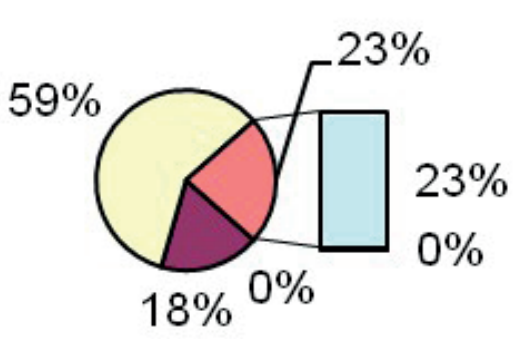

Los clientes expresaron que cuando el gestor no les visita corren el riesgo de que en el tiempo que pierden esperando la visita del gestor para el cobro de la factura de energía, se les genere el corte del servicio y corran con el gasto del cargo de la reconexión que cobra da Distribuidora de electricidad y a la ves al tener que buscar otro punto de recaudo para pagar tienen gastos de transporte y tiempo.

Pregunta 5 ¿Es de su entera confianza la gestión que realiza el gestor?

\section{Es de su entera confianza la gestión que realiza el gestor}

Acerca de la Satisfacción del cliente el $18 \%$ dijo que el servicio era muy bueno, el 59\% lo cataloga como bueno y el $23 \%$ dice que es regular.

Según Sergio A. Pedemonte Mery (2003), todos saben que vivimos en un entorno altamente competitivo, donde muchos hemos sido testigos de las guerras que han desatado algunas empresas por captar clientes, y que han dejado a más de un lesionado. Ahora más que nunca las empresas buscan fórmulas para retener a sus clientes y evitar que estos queden a merced de la competencia.

En este sentido la satisfacción del cliente se transforma en un elemento clave para lograr la retención de los clientes en el largo plazo, pero a menudo las empresas no se ocupan de este factor al no preocuparse de medir cual es el real grado de satisfacción de sus clientes. Suponen que si no reciben quejas, entonces todo marcha a la perfección, y esto es un error, ya es un hecho de que la mayoría de los clientes insatisfechos nunca se queja, simplemente muchos de ellos jamás volverán a adquirir su producto o servicio y se pasarán a la competencia.

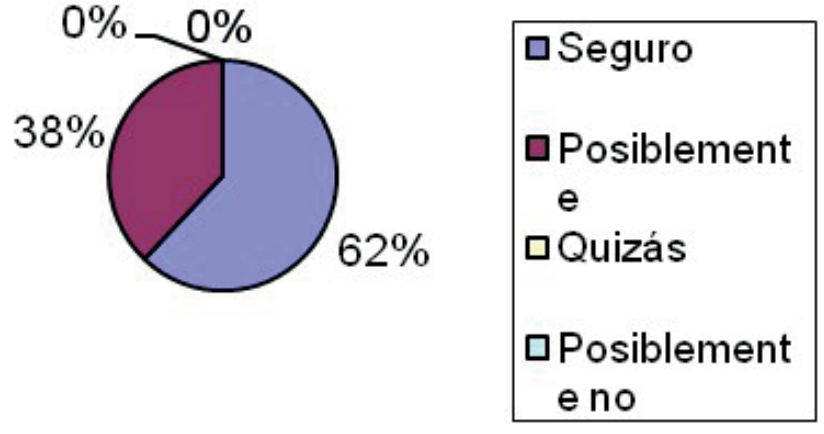

Al consultar acerca de la confianza que sentía al pagar sus facturas a un gestor de cobro y no a la empresa (Disnorte) directamente, el $62 \%$ dijo estar seguro de realizar el pago al gestor y el 38\% dijo dudar un poco del servicio.

La cualidades del gestor deben ser similares al ejecutivo del crédito, porque lo más recomendado es que la misma persona quien se responsabilice de la cobranza, debe tener conocimientos de los créditos otorgados por la empresa. El cobrador debe tener un conocimiento completo de las técnicas de cobranza y saber cómo y cuándo aplicarla. El cobrador debe poseer las siguientes cualidades: honestidad, responsabilidad, integridad, creatividad, iniciativa, paciencia entre otros. 
Hasta la fecha en el departamento de Estelí y sus municipios no hemos tenido incidencias de robos provenientes de gestores asignados a la zona. Cada gestor entrega a la Oficina comercial más cercana el cobro diario realizado, con sus respectivas minutas de depósito y los cupones de las facturas canceladas por lo clientes. Es mínima la ocasión en que un cliente ha llegado a informar a la Distribuidora que ya cancelo a un gestor de ECONORTE y el pago no este registrado en sistema, podemos decir que en un año tenemos un aproximado de 5 incidencias.

Pregunta 6: ¿Es visitado todos los meses por el gestor de cobro?

\section{Es visitado todos los meses por el gestor de cobro}

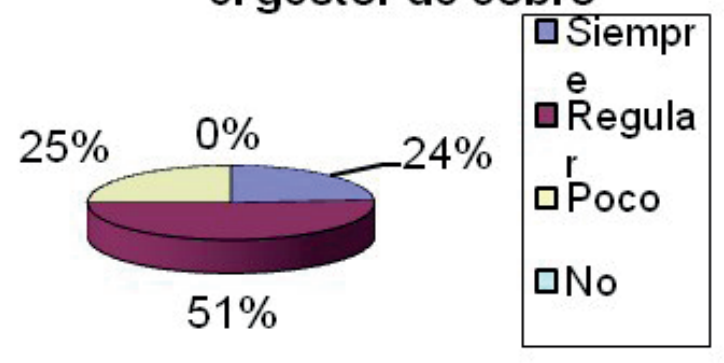

Se consultó también si todos los meses era visitado por el gestor de cobro en su propiedad y el $24 \%$ informa que siempre es visitado, el $51 \%$ dijo que regularmente y el restante $25 \%$ dijo que pocas veces.

Se demuestra la falta de gestión de cobro casa a casa ya que al consultar a los clientes si eran visitados mensualmente la mayoría dijo que regularmente, por lo que no están cumpliendo con el contrato que tienen con la Distribuidora del Norte el cual específicamente les orienta la gestión de cobro de cuentas por cobrar casa a casa. El cliente espera mes a mes al gestor para realizar sus pagos y claramente se nota en el desarrollo de la encuesta que no se está dando seguimiento mensual a los clientes, al parecer un mes es visitado y el siguiente no.
Gestión de Cobro: Para analizar la gestión de cobro se aplicaron una encuesta los Gestores de cobro y obteniendo los siguientes resultados:

Al preguntarles si tenían rutas establecidas y cronogramas de visitas me informan que si tienen y que se coordinan con el Distribuidor de Facturas en la zona que le corresponde a cada uno además de dejar los números de teléfonos a los clientes para que les llamen.

¿Les da seguimiento a los clientes mensualmente? Todos los entrevistados respondieron que dan seguimiento cada vez mediante visitas a los clientes de la zona que les corresponde.

Sobre sus metas de cobro, respondieron que tienen metas individuales y las asignadas por la empresa contratante.

En relación a la información a la empresa contratante el monto de lo cobrado y cantidad de facturas recuperadas, afirman que el reporte del cobro diario lo realizan mediante un programa llamado APLICOP (Sistema de aplicación local) el cual está instalado en cada punto fijo de su municipio que luego es ingresado en el sistema de DISNORTE (Distribuidora de electricidad del norte) para aplicar los pagos realizados por los clientes al gestor.

\section{CONCLUSIONES}

Al aplicar los instrumentos como la encuesta y la observación y analizar los resultados, a continuación detallo los datos relevantes en la misma:

Me doy cuenta que según los gestores de cobro de ECONORTE las acciones y estrategias que siguen para la recuperación de la cuentas por cobrar de la empresa DISNORTE son las correctas y se le esta dando el debido seguimiento a los clientes, además de estar en comunicación con los Distribuidores de Facturas para coordinar el cobro de las mismas. 
Según la encuesta realizada a los clientes lo anterior no es del todo efectivo ya que no se le esta realizando la visita mensualmente por lo que al parecer la gestión no es la correcta.

Se analizaron las metas de cobro de la empresa ECONORTE asignadas por DISNORTE en el 2011 por mes y se encontró que solamente en el mes de Abril, Mayo, Junio y Agosto -11 la empresa logro cumplir con las metas asignadas con $100 \%$ o un poco más.

El resto de los meses no logro el cumplimiento de las metas, llegando a quedar hasta con $84 \%$ de recuperación.

Lo anterior refleja que cada gestor realiza el cobro a su conveniencia y comodidad, quedándose nada más la recuperación dentro del punto fijo y en la zona urbana de su municipio.

Adjunto en anexos las metas asignadas mensualmente por la Distribuidora y la recuperación presentada por ECONORTE.

\section{BIBLIOGRAFÍA}

Beaugond,R.(1997) Aspectos principales a controlar en la administración del crédito. Recuperado el 4 de diciembre del 2012. Sitio web: http://www. monografias.com/trabajos32/gerencia-creditos/ gerencia-creditos3.

Carh, Pos. (2007). Estrategia-Cobranzas. Recuperado el día 12 de septiembre del 2012. Sitio web: http:// www.cash-pot.com.ar/gestion-de-cobranzas.aspx

Ettinger, R, P. (2000). Crédito y Cobranza. Disponible en el sitio internet http://www.monografias. com/trabajos80/ejecutivo-cobranza/ejecutivocobranza2.

Figueros, S ( 2003) Formulas-para-dar-a-conocer-tunegocio/Recuperado el día 3 de Enero del 2013. Sitio web: www.salvadorfigueros.com/3 Montaño, A. (1987). Administración de la Cobranza.
Editorial Trillas. Venezuela. Recuperado el 25 de noviembre. Sitio web: http://www.monografias. com/trabajos80/ejecutivo-cobranza/ejecutivocobranza2.

SERVIMEDIA. (2008). Expertos recomiendan combatir la morosidad con diferentes estrategias de gestión de cobro según el cliente. Recuperado el día 8 de noviembre del 2012. Sitio web: www.eleconomista.es/economia/ noticias/424856/03/08/Expertos-

Shuktz, J. W. (1987). Crédito y Cobranza. México: Editorial Hispanoamérica.

Sibila Customer Intelligence ${ }^{\mathrm{TM}}$. Solución avanzada para marketing y ventas (2011) Recuperado el 02 de Enero-2013. Sitio web: www.sibilaintelligence. com/soluciones/ 